SCIREA Journal of Mathematics http://www.scirea.org/journal/Mathematics

November 9, 2021

Volume 6, Issue 5, October 2021

\title{
Comparison principle to the infinity Laplacian equation with lower term
}

\section{Cuicui Li, Fang Liu*}

School of Mathematics and Statistics, Nanjing University of Science and Technology, Nanjing 210094, Jiangsu, People's Republic of China

Email: licui1121@njust.edu.cn (Cuicui Li),sdqdlf78@sina.com (Fang Liu)

*Corresponding author

This work was supported by National Natural Science Foundation of China(No.11501292)

\section{Abstract}

In this paper, we establish the comparison principles via the perturbation method for the equation $\Delta_{\infty}^{h} u+A(D u)=f(x, u)$ in $\Omega$, where $\Delta_{\infty}^{h} u=|D u|^{h-3}\left\langle D^{2} u D u, D u\right\rangle$ is a highly degenerate and $\mathrm{h}^{-}$homogeneous operator associated with the infinity Laplacian. Based on the comparison principle, we obtain the uniqueness of the viscosity solution to the Dirichlet problem

$$
\left\{\begin{array}{cc}
\Delta_{\infty}^{h} u+A(D u)=f(x, u), & \text { in } \Omega, \\
u=g, & \text { on } \partial \Omega,
\end{array}\right.
$$

where $g \in C(\partial \Omega)$. During this procedure, we also establish a stability result of the viscosity solution to the inhomogeneous equation. 
Keywords: infinity Laplacian; viscosity solutions; comparison principle; stability.

2010 Mathematic Subject Classification. 35D40; 35B51; 34D20; 35J70.

\section{Introduction}

In this paper, we consider the following inhomogeneous problems for $g \in C(\partial \Omega)$ :

$$
\left\{\begin{array}{lr}
\Delta_{\infty}^{h} u+A(D u)=f(x, u), & \text { in } \Omega, \\
u=g, & \text { on } \partial \Omega,
\end{array}\right.
$$

where $\Omega$ be a bounded domain in $\mathbb{R}^{n}(n \geq 2), A(p): \mathbb{R}^{n} \rightarrow \mathbb{R}$ is continuous and homogeneous of degree $h, f(x, t) \in C(\Omega \times \mathbb{R})$ is non-decreasing in the second variable $t, \Delta_{\infty}^{h}$ is a highly degenerate elliptic operator given by

$$
\Delta_{\infty}^{h} u=|D u|^{h-3} \sum_{i, j=1}^{n} u_{i} u_{j} u_{i j}, \quad h>1 .
$$

Notice that the operator $\Delta_{\infty}^{h}$ is not in divergence form. Hence, the notion of the solution is understood here in the viscosity sense introduced by Crandall, Lions [9], and Crandall, Evans, Lions [10].

For the case $h=1, \Delta_{\infty}^{h} u$ is the 1-homogeneous normalized infinity-Laplacian operator,

$$
\Delta_{\infty}^{N} u:=|D u|^{-2}\left\langle D^{2} u D u, D u\right\rangle .
$$

And there is a "tug-of-war" game approach to the following normalized infinity Laplacian Dirichlet problem mentioned in [23],

$$
\begin{cases}\Delta_{\infty}^{N} u=f(x), & \text { in } \Omega, \\ u=g, & \text { on } \partial \Omega .\end{cases}
$$

In [19], Lu and Wang gave a different proof from the PDEs perspective for the existence and uniqueness of the viscosity solution of the problem (1.2). One can see [4, 15, 18, 22, 24], etc. for more about stochastic approach related to the normalized $\infty$-Laplacian operator.

For the case $h=3, \Delta_{\infty}^{h} u$ is the 3-homogeneous infinity-Laplacian operator, 


$$
\Delta_{\infty} u:=|D u|^{2} \Delta_{\infty}^{N} u
$$

The infinity Laplacian $\Delta_{\infty}$ was first introduced by Aronsson [2] in connection with the geometric problem of finding the so-called absolutely minimizing Lipschitz extension. For more properties of the infinity harmonic functions (the viscosity solution to $\Delta_{\infty} u=0$ ), one can see the works of Crandall [7], Crandall, Evans and Gariepy [8], Aronsson, Crandall and Juutinen [3], and the references therein.

For other $h$, we have $\Delta_{\infty}^{h} u=|D u|^{h-3} \Delta_{\infty} u=|D u|^{h-1} \Delta_{\infty}^{N} u$.

In [20], Lu and Wang proved the comparison principle and existence of the viscosity solution to the infinity Laplace equation

$$
\Delta_{\infty} u=f(x)
$$

under Dirichlet condition. Moreover, they also obtained the stability of the viscosity solutions to $\Delta_{\infty} u=f(x)$. It should be pointed out that they constructed a counterexample to show that the uniqueness does not hold if the inhomogeneous term $f(x)$ changes its sign. A counterexample is given for the normalized infinity Laplacian equation

$$
\Delta_{\infty}^{N} u=f(x)
$$

in [23]. In [5], Bhattacharya and Mohammed considered the comparison principle, existence and nonexistence of the viscosity solutions to a general infinity Laplacian equation with zero order term

$$
\Delta_{\infty} u=f(x, u)
$$

with Dirichlet condition. In [17], Liu and Yang established the existence of the viscosity solution to the Dirichlet problem of the $h$-infinity Laplacian

$$
\Delta_{\infty}^{h} u=f(x) .
$$

In this paper, we are interested in the problem (1.1) not only because it is strong degenerate and nonlinear but also it has many applications such as in optimal mass transportation [12, 14], mathematical finance [21], digital image processing [1, 6, 13], etc.

Now we state our main results as follows.

Theorem 1.1 (Comparison principle). Suppose that $f(x, t) \in C(\Omega \times \mathbb{R})$ is positive (negative) 
and non-decreasing in $t$. Assume that $u \in C(\bar{\Omega})$ and $v \in C(\bar{\Omega})$ satisfy

$$
\Delta_{\infty}^{h} u+A(D u) \geq f(x, u), \quad x \in \Omega
$$

and

$$
\Delta_{\infty}^{h} v+A(D v) \leq f(x, v), x \in \Omega
$$

in the viscosity sense. If $u \leq v$ on $\partial \Omega$, then $u \leq v$ in $\Omega$.

Due to the strong degeneracy of the operator $\Delta_{\infty}^{h}$, we adopt the perturbation method based on the viscosity solutions theory to establish the comparison principle. With the comparison principle in hand, the uniqueness of the Dirichlet problem (1.1) follows immediately.

Theorem 1.2 (uniqueness). If $f(x, t) \in C(\Omega \times \mathbb{R})$ is positive (negative) and non-decreasing in $t$, then there exists at most one viscosity solution to the Dirichlet problem (1.1).

Based on the double variables argument, we can give the following stability result of the viscosity solutions.

Theorem 1.3 (stability). Let $\left\{f_{k}\right\}_{k=1}^{\infty}$ be a sequence of non-negative functions in $C(\Omega)$ such that $f_{k} \rightarrow f$ locally uniformly in $\Omega$ for some $f \in C(\Omega)$. Suppose that for each positive integer $k, u_{k} \in C(\bar{\Omega})$ is a viscosity solution to the problem

$$
\begin{cases}\Delta_{\infty}^{h} u=f_{k}, & \text { in } \Omega, \\ u_{k}=g & \text { on } \partial \Omega\end{cases}
$$

such that $u_{0} \leq u_{k} \leq u_{\infty}$ in $\bar{\Omega}$, for some functions $u_{0}$ and $u_{\infty}$ in $C(\bar{\Omega})$, with $u_{0}=u_{\infty}=\mathrm{g}$ on $\partial \Omega$. Then $\left\{u_{k}\right\}$ has a subsequence that converges locally uniformly in $\Omega$ to a viscosity solution $u \in C(\bar{\Omega})$ to the problem 


$$
\begin{cases}\Delta_{\infty}^{h} u=f_{k}, & \text { in } \Omega, \\ u_{k}=g, & \text { on } \partial \Omega .\end{cases}
$$

The paper is organized as follows. In Section 2, we state the definition of viscosity solutions to the Dirichlet problem (1.1). In Section 3, we prove the comparison principle to $\Delta_{\infty}^{h} u+$ $A(D u)=f(x, u)$, based on the double variables method. And we also use the perturbation method to prove the stability of the viscosity solution.

\section{Definition of viscosity solutions}

In this section, we first introduce the definition of the viscosity solutions to the problem (1.1), and then establish the comparison principles via the perturbation method for the equation

$$
\Delta_{\infty}^{h} u+A(D u)=f(x, u), \quad \text { in } \Omega
$$

Since the operator has no divergence structure, we define the viscosity solution by the semicontinuous extension. See for example $[11,16,19]$, etc. We remark that the singularity is removable for $h>1$. Then for $F_{h}: \mathbb{S} \times\left(\mathbb{R}^{n}\{0\}\right) \rightarrow \mathbb{R}$ and

$$
F_{h}(M, p):=|p|^{h-3}(M p) \cdot p+A(p)
$$

where $\mathbb{S}$ denotes the set of $n \times n$ real symmetric matrices, we can rewrite the equation (2.1) as

$$
F_{h}\left(D^{2} u, D u\right)=f(x, u), \quad x \in \Omega .
$$

Due to $A(p) \in C\left(\mathbb{R}^{n}\right)$ is homogeneous of degree $h$, we obtain $A(0)=0$. Since $h>1$, then we have $\lim _{p \rightarrow 0} F_{h}(M, p)=0$ for arbitrary $M \in \mathbb{S}$. That is, the operator $\Delta_{\infty}^{h}$ is continuous for $h>1$. Therefore, we can define the continuous extension of $F_{h}$ as follows,

$$
\overline{F_{h}}(M, p):= \begin{cases}F_{h}(M, p), & \text { if } p \neq 0, \\ 0, & \text { if } p=0 .\end{cases}
$$

Now we state the definition of viscosity solutions to the problem (1.1).

Definition 2.1. Suppose that $u: \bar{\Omega} \rightarrow \mathbb{R}$ is an upper semi-continuous function and $u \leqslant g$ on $\partial \Omega$. If for every $x_{0} \in \Omega$ and $\varphi \in C^{2}(\Omega)$ such that $u\left(x_{0}\right)=\varphi\left(x_{0}\right)$ and $u(x) \leqslant \varphi(x)$ for all $x \in \Omega$ near $x_{0}$, there holds 


$$
\bar{F}_{h}\left(D^{2} \varphi\left(x_{0}\right), D \varphi\left(x_{0}\right)\right) \geqslant f\left(x_{0}, \varphi\left(x_{0}\right)\right) .
$$

Then we say that $u$ is a viscosity subsolution to (1.1).

Similarly, suppose that $u: \bar{\Omega} \rightarrow \mathbb{R}$ is a lower semi-continuous function and $u \geqslant g$ on $\partial \Omega$. If for every $x_{0} \in \Omega$ and $\varphi \in C^{2}(\Omega)$ such that $u\left(x_{0}\right)=\varphi\left(x_{0}\right)$ and $u(x) \geqslant \varphi(x)$ for all $x \in$ $\Omega$ near $x_{0}$, there holds

$$
\bar{F}_{h}\left(D^{2} \varphi\left(x_{0}\right), D \varphi\left(x_{0}\right)\right) \leqslant f\left(x_{0}, \varphi\left(x_{0}\right)\right) .
$$

Then we say that $u$ is a viscosity supersolution to (1.1).

A function $u \in C(\Omega)$ is a viscosity solution to (1.1) in $\Omega$ if it is both a viscosity subsolution and viscosity supersolution of (1.1).

We can also use sub-jets and super-jets (see [11]) to define the viscosity subsolution and the viscosity supersolution equivalently. Now we first recall the definition of sub-jets and superjets.

The second order super-jet of upper semi-continuous function $u$ at $x_{0} \in \Omega$ is the set

$$
\mathcal{J}^{2,+} u\left(x_{0}\right)=\left\{\left(D \varphi\left(x_{0}\right), D^{2} \varphi\left(x_{0}\right)\right): \varphi \in C^{2}(\Omega) \text { and } u-\varphi \text { has a local maximum at } x_{0}\right\},
$$

and its closure is

$$
\begin{gathered}
\bar{J}^{2,+} u\left(x_{0}\right)=\left\{(p, M) \in \mathbb{R}^{n} \times \mathbb{S}: \exists\left(x_{i}, p_{i}, M_{i}\right) \in \Omega \times \mathbb{R} \times \mathbb{S}\right. \text { sunch thatt } \\
\left.\left(p_{i}, M_{i}\right) \in \mathcal{J}^{2,+} u\left(x_{i}\right) \text { and }\left(x_{i}, p_{i}, M_{i}\right) \rightarrow\left(x_{0}, p, M\right)\right\}
\end{gathered}
$$

Similarly, the second order sub-jet of lower semi-continuous function $u$ at $x_{0} \in \Omega$ is the set

$$
\mathcal{J}^{2,-} u\left(x_{0}\right)=\left\{\left(D \varphi\left(x_{0}\right), D^{2} \varphi\left(x_{0}\right)\right): \varphi \in C^{2}(\Omega) \text { and } u-\varphi \text { has a local minimum at } x_{0}\right\}
$$

and its closure is

$$
\begin{gathered}
\bar{J}^{2,-} u\left(x_{0}\right)=\left\{(p, M) \in \mathbb{R}^{n} \times \mathbb{S}: \exists\left(x_{i}, p_{i}, M_{i}\right) \in \Omega \times \mathbb{R} \times \mathbb{S}\right. \text { sunch thatt } \\
\left.\left(p_{i}, M_{i}\right) \in \mathcal{J}^{2,-} u\left(x_{i}\right) \text { and }\left(x_{i}, p_{i}, M_{i}\right) \rightarrow\left(x_{0}, p, M\right)\right\}
\end{gathered}
$$

Definition 2.2. We say that $u \in C(\Omega)$ is a viscosity subsolution to (2.1) if 


$$
\bar{F}_{h}(M, p) \geqslant f\left(x_{0}, u\left(x_{0}\right)\right), \quad \forall(p, M) \in \overline{\mathcal{J}}^{2,+} u\left(x_{0}\right), \forall x_{0} \in \Omega .
$$

Similarly, we say that $u \in C(\Omega)$ is a viscosity supersolution to (2.1) if

$$
\bar{F}_{h}(M, p) \leqslant f\left(x_{0}, u\left(x_{0}\right)\right), \quad \forall(p, M) \in \overline{\mathcal{J}}^{2,-} u\left(x_{0}\right), \forall x_{0} \in \Omega .
$$

\section{Comparison principle}

In this section, we first prove the comparison principle by the perturbation method based on the viscosity solutions theory, and then establish a stability result.

Now we recall the maximum principle for infinity harmonic functions which can be deduced from Harnack's inequality ( see for example $[3,7]$ ).

Lemma 3.1 Let $u \in C(\bar{\Omega})$ satisfy $\Delta_{\infty} u \geqslant 0$ in the viscosity sense. Then

$$
\sup _{\Omega} u=\sup _{\partial \Omega} u .
$$

Moreover, the supremum occurs only on the boundary $\partial \Omega$ unless $u$ is a constant.

Next, we use the double variables method to prove the comparison principle.

Proof of Theorem 1.1. We consider the case for $f(x, t)$ is positive. The case for $f(x, t)$ is similar and we leave it to the reader.

We define

$$
u_{\varepsilon}:=u+\varepsilon\left(u-\sup _{\partial \Omega} u .\right), \quad \varepsilon>0 .
$$

By Lemma 3.1, we have $u \leqslant \sup u$. and $u_{\varepsilon} \leqslant u$ in $\Omega$. Since $f(x, t)>0$ in $\Omega \times \mathbb{R}$ and $\partial \Omega$ $A(p) \in C\left(\mathbb{R}^{n}, \mathbb{R}\right)$ is homogeneous of degree $h$, it is easy to verify

$$
\Delta_{\infty}^{h} u_{\varepsilon}+A\left(D u_{\varepsilon}\right)=(1+\varepsilon)^{h}\left[\Delta_{\infty}^{h} u+A(D u)\right] \geqslant(1+\varepsilon)^{h} f(x, u) \geqslant(1+\varepsilon)^{h} f\left(x, u_{\varepsilon}\right) \geqslant f\left(x, u_{\varepsilon}\right),
$$

that is, $u_{\varepsilon}$ is a viscosity subsolution to the equation (2.1).

We claim that $u_{\varepsilon} \leqslant v$ in $\Omega$. We argue by contradiction. Suppose that $u_{\varepsilon}>v$ somewhere in $\Omega$. 
Set

$$
M=\sup _{\Omega}\left(u_{\varepsilon}-v\right)=u_{\varepsilon}\left(x_{0}\right)-v\left(x_{0}\right)>0
$$

Using the arguments in [11], we double the variables

$$
w_{j}(x, y)=u_{\varepsilon}(x)-v(y)-j|x-y|^{4} / 4,(x, y) \in \Omega \times \Omega, j=1,2, \cdots .
$$

We denote the maximum point of $w_{j}$ over $\bar{\Omega} \times \bar{\Omega}$ by $\left(x_{j}, y_{j}\right)$. According to Proposition 3.7 in [11], we have

$$
\lim _{j \rightarrow \infty} M_{j}=\lim _{j \rightarrow \infty}\left(u_{\varepsilon}\left(x_{j}\right)-v\left(y_{j}\right)-j\left|x_{j}-y_{j}\right|^{4} / 4\right)=M
$$

and

$$
\lim _{j \rightarrow \infty} j\left|x_{j}-y_{j}\right|^{4} / 4=0
$$

It is obvious $x_{j} \rightarrow x_{0}, y_{j} \rightarrow x_{0}$ as $j \rightarrow \infty$. Since $M>0 \geqslant \sup \left(u_{\varepsilon}-v\right)$, there is an open set $\partial \Omega$

$\Omega_{0}$ such that $x_{0}, x_{j}$ and $y_{j} \in \Omega_{0} \subseteq \Omega$ for $j \rightarrow \infty$.

Set

$$
\varphi(x)=j\left|x-y_{j}\right|^{4} / 4, \quad \phi(y)=-j\left|x_{j}-y\right|^{4} / 4 .
$$

It is clear that the functions $u_{\varepsilon}-\varphi$ and $v-\phi$ have a local maximum at $x_{j}$ and a local minimum at $y_{j}$ respectively. We consider the two cases: either $x_{j} \neq y_{j}$ or $x_{j}=y_{j}$ for $j \rightarrow \infty$.

Case 1: If $x_{j}=y_{j}$, we have $D \varphi\left(x_{j}\right)=0$ and $D^{2} \varphi\left(x_{j}\right)=0$. Since $A(p) \in C\left(\mathbb{R}^{n}\right)$ is homogeneous of degree $h$, we get $A(0)=0$. And since $u_{\varepsilon}$ is a viscosity subsolution, we have

$$
f\left(x_{j}, \varphi\left(x_{j}\right)\right)=f\left(x_{j}, u_{\varepsilon}\left(x_{j}\right)\right) \leqslant 0
$$

which is contradictory to $f>0$ in $\Omega$.

Case 2: If $x_{j} \neq y_{j}$, we shall use jets and maximum principle for semi-continuous functions, see [11]. There exist $n \times n$ symmetric matrices $X_{j}$ and $Y_{j}$ such that $Y_{j}-X_{j} \geqslant 0$ and 


$$
\left(p_{j}, X_{j}\right) \in \bar{\jmath}^{2,+} u_{\varepsilon}\left(x_{j}\right), \quad\left(p_{j}, Y_{j}\right) \in \bar{\jmath}^{2,-} v\left(y_{j}\right),
$$

where $p_{j}=j\left|x_{j}-y_{j}\right|^{2}\left(x_{j}-y_{j}\right)$. Again since $\Delta_{\infty}^{h} u_{\varepsilon}+A\left(D u_{\varepsilon}\right) \geqslant(1+\varepsilon)^{h} f\left(x, u_{\varepsilon}\right) \geqslant f\left(x, u_{\varepsilon}\right)$ and $\Delta_{\infty}^{h} v+A(D v) \leqslant f(x, v)$ in the viscosity sense, we have

$$
\begin{aligned}
0 & \leqslant\left|p_{j}\right|^{h-3}\left\langle X_{j} p_{j}, p_{j}\right\rangle+A\left(p_{j}\right)-(1+\varepsilon)^{h} f\left(x_{j}, u_{\varepsilon}\left(x_{j}\right)\right) \\
& \leqslant\left|p_{j}\right|^{h-3}\left\langle Y_{j} p_{j}, p_{j}\right\rangle+A\left(p_{j}\right)-f\left(y_{j}, v\left(y_{j}\right)\right)+f\left(y_{j}, v\left(y_{j}\right)\right)(1+\varepsilon)^{h} f\left(x_{j}, u_{\varepsilon}\left(x_{j}\right)\right) \\
& \leqslant f\left(y_{j}, v\left(y_{j}\right)\right)-(1+\varepsilon)^{h} f\left(x_{j}, u_{\varepsilon}\left(x_{j}\right)\right)
\end{aligned}
$$

where we have used $Y_{j}-X_{j} \geqslant 0$. Letting $j \rightarrow \infty$, we get

$$
f\left(x_{0}, v\left(x_{0}\right)\right)-(1+\varepsilon)^{h} f\left(x_{0}, u_{\varepsilon}\left(x_{0}\right)\right) \geqslant 0 .
$$

Since $f(x, t)$ is non-decreasing in $t$ and $u_{\varepsilon}\left(x_{0}\right)>v\left(x_{0}\right)$, we have $f\left(x_{0}, u_{\varepsilon}\left(x_{0}\right)\right) \geqslant$ $f\left(x_{0}, v\left(x_{0}\right)\right)$ which contradicts to (3.3). Hence, we have $u_{\varepsilon} \leqslant v$ in $\Omega$. Letting $\varepsilon \rightarrow 0$, we get $u \leqslant v$ in $\Omega$.

Now, we prove the stability of the problem (1.3).

Proof of Theorem 1.3. Set $M:=\sup _{\Omega} u_{\infty}-\inf _{\Omega} u_{0}$. Clearly, we have $\sup _{\Omega} u_{k}-\inf _{\Omega} u_{k} \leq M$, for every $k=1,2, \cdots$. Let $\Omega_{0}$ be any compact subset of $\Omega$ and $d:=\operatorname{dist}\left(\Omega_{0}, \partial \Omega\right)$. We take $R>0$ such that $4 R<d$. Since $\Delta_{\infty}^{h} u_{k} \geqslant 0$ in $\Omega$, we have $\Delta_{\infty} u_{k} \geqslant 0$ in $\Omega$. By Lemma 2.9 of [3], we get

$$
\left|u_{k}(x)-u_{k}(y)\right| \leqslant M \frac{|x-y|}{R}, \quad \forall z \in \Omega_{0}, x, y \in B_{R / 2}(z) .
$$

By compactness, we get $\left\{u_{k}\right\}$ is equicontinuous in $\Omega_{0}$. On taking an exhaustion of $\Omega$ by subdomains compactly contained in $\Omega$, we apply the standard method of Cantor diagonalization to extract a subsequence of $\left\{u_{k}\right\}$ that converges uniformly on compact subsets of $\Omega$. For simplicity we will continue to denote such subsequence by $\left\{u_{k}\right\}$. Set

$$
u(x):=\lim _{k \rightarrow \infty} u_{k}(x), \quad x \in \Omega
$$

We extend this definition to the closure $\bar{\Omega}$ by defining $u=g$ on $\partial \Omega$. By the assumption, we 
have $u_{0} \leqslant u \leqslant u_{\infty}$ in $\bar{\Omega}$. This means that $u \in C(\bar{\Omega})$

Next, we show that $\Delta_{\infty}^{h} u=f$ in the viscosity sense. Suppose that $\varphi \in C^{2}(\Omega)$ and $u-\varphi$ has a local maximum at some $x_{0} \in \Omega$, i.e.

$$
u(x)-\varphi(x) \leqslant u\left(x_{0}\right)-\varphi\left(x_{0}\right), \quad x \in B_{r}\left(x_{0}\right) \subseteq \Omega
$$

for some $r>0$. Suppose that $x_{k}$ is a point of maximum of

$$
u_{k}(x)-\left(\varphi(x)+\frac{\varepsilon}{2}\left|x-x_{0}\right|^{2}\right), \quad \varepsilon>0, x \in \bar{B}_{r}\left(x_{0}\right) .
$$

Particularly,

$$
u_{k}\left(x_{k}\right)-\left(\varphi\left(x_{k}\right)+\frac{\varepsilon}{2}\left|x_{k}-x_{0}\right|^{2}\right) \geqslant u_{k}\left(x_{0}\right)-\varphi\left(x_{0}\right)
$$

Since $x_{k} \in \bar{B}_{r}\left(x_{0}\right)$, by passing to a subsequence, $x_{k} \rightarrow \hat{x}$, for some $\hat{x} \in \bar{B}_{r}\left(x_{0}\right)$. Letting $k \rightarrow \infty$ in (3.4), we have

$$
u(\hat{x})-\left(\varphi(\hat{x})+\frac{\varepsilon}{2}\left|\hat{x}-x_{0}\right|^{2}\right) \geqslant u\left(x_{0}\right)-\varphi\left(x_{0}\right)
$$

i.e.

$$
\frac{\varepsilon}{2}\left|\hat{x}-x_{0}\right|^{2} \leqslant u(\hat{x})-\varphi(\hat{x})-\left(u\left(x_{0}\right)-\varphi\left(x_{0}\right)\right) \leqslant 0 .
$$

Then we have $\hat{x}=x_{0}$ Thus, $x_{k} \in B_{r / 2}\left(x_{0}\right)$ for sufficiently large $k$. Since $u_{k}$ is a viscosity subsolution and $x_{k}$ is a point of local maximum of $u_{k}(x)-\left(\varphi(x)+\frac{\varepsilon}{2}\left|x-x_{0}\right|^{2}\right)$ in $B_{r}\left(x_{0}\right)$, we have

$$
\Delta_{\infty}^{h} \varphi\left(x_{k}\right)+O(\varepsilon) \geqslant f_{k}\left(x_{k}\right)
$$

Taking the limit in (3.5) and recalling that $f_{k} \rightarrow f$ locally uniformly in $\Omega$, we find that

$$
\Delta_{\infty}^{h} \varphi\left(x_{0}\right)+O(\varepsilon) \geqslant f\left(x_{0}\right)
$$

Letting $\varepsilon \rightarrow 0$, we have $\Delta_{\infty}^{h} u \geqslant f$ in the viscosity sense. Similarly, we can prove that $u$ is a viscosity supersolution. 


\section{References}

[1] E. Abderrahim, D. Xavier, L. Zakariaa and L. Olivier, Nonlocal infinity Laplacian equation on graphs with applications in image processing and machine learning, Mathematics and Computers in Simulation, 102(2014), 153-163.

[2] G. Aronsson, Extension of functions satisfying Lipschitz conditions, Ark. Mat. 6(1967), 551-561.

[3] G. Aronsson, M. G. Crandall, P. Juutinen, A tour of the theory of absolutely minimizing functions, Bull. Amer. Math. Soc. 41(2004), 439-505.

[4] S. Armstrong, C. Smart, An easy proof Jensen's theorem on the uniqueness of infinity harmonic functions. Calc. Var. Partial Differ. Equ. 37(2010), 381-384.

[5] T. Bhattacharya, A. Mohammed, On solutions to Dirichlet problems involving the infinity-Laplacian, Adv. Calc. Var. 4(2011), 445-487.

[6] V. Caselles, J. M. Morel and C. Sbert, An axiomatic approach to image interpolation, IEEE Trans. Image Process, 7(1998), 376-386.

[7] M. G. Crandall, A visit with the $\infty$-Laplace equation, in Calculus of Variations and Nonlinear Partial Differential Equations, Lecture Notes in Math. 1927, pp. 75-122, Springer, Berlin, 2008.

[8] M. G. Crandall, L. C. Evans and R. F. Gariepy, Optimal Lipschitz extensions and the infinity-Laplacian, Calc. Var. Partial Differ. Equ. 13(2001), 123-139.

[9] M. G. Crandall, P. L. Lions, Viscosity solutions and Hamilton-Jacobi equations. Trans. Am. Math. Soc. 277(1983), 1-42.

[10] M. G. Crandall, L. C. Evans, P. L. Lions, Some properties of viscosity solutions of Hamilton-Jacobi equations. Trans. Am. Math. Soc. 282(1984), 487-502.

[11] M. G. Crandall, H. Ishii, P. L. Lions, User's guide to viscosity solutions of second order partial differential equations, Bull. Amer. Math. Soc. (N. S.) 27(1992), 1-67.

[12] L. C. Evansand W.Gangbo, Differential equations methods for the Monge-Kantorovich mass transfer problem, Mem. Amer. Math. Soc., 137 (1999) no.653, viii+66 pp.

[13] A. Elmoataz, M. Toutain, D. Tenbrinck, On the p-Laplacian and $\infty$-Laplacian on graphs with applications in image and data processing, SIAM J. Imaging Sciences, 8(4) (2015), 2412-2451.

[14] J. Garcia-Azorero, J. J. Manfredi, I. Peral and J. D. Rossi, The Neumann problem for the $\infty$-Laplacian and the Monge-Kantorovich mass transfer problem, Nonlinear Analysis: Theory Methods \& Applications, 66 (2007), 349-366. 
[15] F. Liu, An inhomogeneous evolution equation involving the normalized infinity Laplacian with a transport term, Commun Pure Appl Anal, 17(6) (2018), 2395-2421.

[16] F. Liu, F. Jiang, Parabolic Biased Infinity Laplacian Equation Related to the Biased Tugof-War, Advanced Nonlinear Studies, 19(1) (2019), 89-112.

[17] F. Liu, X.P. Yang, Solutions to an inhomogeneous equation involving infinity-Laplacian, Nonlinear Analysis: Theory, Methods \& Applications, 75 (2012), 5693-5701.

[18] R. López-Soriano, J. C. Navarro-Climent and J. D. Rossi, The infinity Laplacian with a transport term. J. Math. Anal. Appl. 398(2013), 752-765.

[19] G. Lu, P. Wang, A PDE perspective of the normalized infinity Laplacian, Comm. Part. Diff. Eqns. 33(10) (2008), 1788-1817.

[20] G. Lu, P. Wang, Inhomogeneous infinity Laplace equation. Adv. Math. 217(2008), 18381868.

[21] K. Nyström, M. Parviainen, Tug-of-war, market manipulation, and option pricing. Math. Finance, 27(2017), 279-312.

[22] Y. Peres, G. Pete, S. Somersille, Biased tug-of-war, the biased infinity Laplacian, and comparison with exponential cones, Calc. Var. PDE. 38(3-4) (2010), 541-564.

[23] Y. Peres, O. Schramm, S. Sheffield, D. Wilson, Tug-of-war and the infinity Laplacian, J. Amer. Math. Soc. 22(1) (2009), 167-210.

[24] J. D. Rossi, Tug-of-war games and PDEs, Proc. Roy. Soc. Edinburgh Sect. A 141(2) (2011), 319-369. 